\title{
A Mathematical Model of The Vertical Movement of the Free Water Level in Fractured Reservoirs
}

\author{
NIELSEN, Birger, COWI Consulting Engineers and Planners AS, Denmark
}

Paper presented at the 5th European Conference on the Mathematics of Oil Recovery, Leoben, Austria, 3-6 Sept. 1996

\begin{abstract}
This paper describes a mathematical model of the vertical movement of the free water level in a fractured reservoir, where the primary recovery is governed by spontaneous water imbibition.- The derived mathematical model is based on a diffusion description of the water imbibition process - which has previously been described and has demonstrated a good modeling ability of laboratory experiments. A variable boundary condition is coupled to a simple material balance equation for a rising water level in a fracture/matrix system. The resulting equations form an infinite set of ordinary first order differential equations which can be solved numerically. Analytic solutions to the diffusion model with various boundary conditions are also presented. The free water level model is formulated both for a single matrix block/fracture system and for a system of vertically stacked blocks/fractures. Numerical results are presented for both systems using typical low-permeability chalk data and constant water injection rates. These show that a constant water velocity in the fracture system is found when the imbibition capacity is capable of fully balancing the water injection rate. In case of a vertically stacked block/fracture system, this occurrence will only depend on the size of the underlying matrix volume exposed to water. In conclusion, it is indicated that the velocity of the free water level in a fractured reservoir does not depend on variation in the matrix blocks' capillary forces, but only on the geometry of the fracture system and the applied water injection rates.
\end{abstract}

\section{INTRODUCTION}

The study of large scale phenomena in reservoir physics is mostly done within the framework of reservoir simulation. Though this may give excellent results, basic physical understanding and obvious facts are likely to be overlooked, due to the huge amount of data in- volved in defining a full $3 \mathrm{D}$ simulation model. A simpler mathematical approach which is derived from the basic physics required by the particular study is therefore often needed.

The object of this paper is to derive a mathematical model of a large scale phenomenon, namely the vertical movement of the free water level in a fractured reservoir subjected to water injection. Oil recovery from such fields is governed primarily by spontaneous imbibition of water into the oil saturated rock, and presents one of the most difficult problems in today's numerical simulation models. In these models the imbibition process is described as a matrix/fracture exchange term, where the global fracture network supports the overall flow, and the intermediate tight matrix formation acts as sources or sinks to this flow. Here we shall present a mathematical model that couples a matrix/fracture exchange term to the overall water level in the fractures. This will allow us to investigate the dynamical behavior of the free water level in a reservoir subjected to different fracture geometries, injection rates and capillary forces. The applied model of the water imbibition matrix/fracture exchange term is based on the diffusion approach of Beckner et $a l^{4}$ and Bech et al. ${ }^{2}$. They found that this model is capable of describing both laboratory experiments and long term imbibition tests with good agreement.

In the first section we shall derive the imbibition model in full, in order to clarify assumptions and limitations of the model.

In the second section we will find the analytic solution of the total water uptake in a rectangular matrix block and give examples of solutions with various boundary conditions imposed.

This result is applied in the third section to couple the fracture water level with the saturations in a single matrix block or a system of vertically stacked blocks. The equations found form an infinite set of first order ordinary differential equations, which can be solved nu- 
merically.

In the next section numerical investigations of the model are discussed - using data from low-permeability chalk. A dominant linear behavior is observed and a simple tank model for describing the dynamics is proposed.

Finally the application of the model with respect to reservoir simulation is discussed.

The following limitations and assumptions have been applied in the formulation.

- Only two-phase oil/water system is considered

- Only valid for countercurrent oil/water flow

- Gravity forces are neglected - only capillary forces are considered

- End effects from capillary forces are not taken into account; i.e. a constant diffusion coefficient is assumed

\section{THE IMBIBITION MODEL}

We consider the flow of two immiscible phases; oil and water, through a porous medium. The mass conservation equations for the two phases can be stated as:

$$
\frac{\partial}{\partial t}\left(\Phi \rho_{j} S_{j}\right)+\nabla \cdot\left(\rho_{j} \vec{u}_{j}\right)=q_{j}
$$

We shall assume that there is constant porosity $\Phi$ and that the fluids are incompressible $\left(\rho_{o}, \rho_{w}\right.$ are constants) and that there are no source terms; i.e. $q_{j}=0$. This simplifies equation (1) to the following:

$$
\Phi \frac{\partial S_{j}}{\partial t}+\nabla \cdot \vec{u}_{j}=0
$$

and using the fact $\sum S_{j}=1$, we notice the following relation holds:

$$
\nabla \cdot \vec{u}_{o}=-\nabla \cdot \vec{u}_{w}
$$

The phase velocities are determined by the Darcy equations:

$$
\vec{u}_{j}=-\lambda_{j}\left(\frac{\partial P_{j}}{\partial x}, \frac{\partial P_{j}}{\partial y}, \frac{\partial P_{j}}{\partial z}+g \rho_{j}\right)
$$

where $\lambda$ is the mobility:

$$
\lambda_{j}=\frac{K k_{j}}{\mu_{j}}
$$

The oil phase pressure gradient is determined by the assumption that the oil flow is either cocurrent or countercurrent with the water flow; i.e.

$$
\vec{u}_{o}=\epsilon \vec{u}_{w} \quad(\epsilon=1 \text { or } \epsilon=-1)
$$

Inserting this assumption into equation (3) yields

$$
(1+\epsilon) \nabla \cdot \vec{u}_{w}=0
$$

For $\epsilon=-1$ this condition is always fulfilled. For $\epsilon \neq$ -1 we have $\nabla \cdot \vec{u}_{w}=0$, imposing a stationary water flow constraint. Thus only a countercurrent flow can be described adequately; i.e. we set $\epsilon \equiv-1$. $\dagger$

The oil phase pressure gradient may now be found from equation (4).

$$
\begin{aligned}
& \frac{\partial P_{o}}{\partial x}=-\frac{\lambda_{w}}{\lambda_{o}} \frac{\partial P_{w}}{\partial x} \quad \ldots \ldots \ldots \ldots \ldots . . . \cdots \\
& \frac{\partial P_{o}}{\partial y}=-\frac{\lambda_{w}}{\lambda_{o}} \frac{\partial P_{w}}{\partial y} \quad \ldots \ldots \ldots \ldots \ldots \\
& \frac{\partial P_{o}}{\partial z}=-\frac{\lambda_{w}}{\lambda_{o}} \frac{\partial P_{w}}{\partial z}-\left(\frac{\lambda_{w}}{\lambda_{o}} \rho_{w}+\rho_{o}\right) g
\end{aligned}
$$

We introduce the capillary pressure $P_{c}\left(S_{w}\right)=P_{o}-P_{w}$ as a function of the water saturation $S_{w}$ only and compute the water phase pressure gradient:

$$
\begin{aligned}
& \frac{\partial P_{w}}{\partial x}=\frac{\partial P_{o}}{\partial x}-\frac{\partial P_{c}}{\partial x}=-\frac{\lambda_{w}}{\lambda_{o}} \frac{\partial P_{w}}{\partial x}-\frac{\partial S_{w}}{\partial x} \frac{d P_{c}}{d S_{w}} \Rightarrow \\
& \left.\left(1+\frac{\lambda_{w}}{\lambda_{o}}\right) \frac{\partial P_{w}}{\partial x}=-\frac{\partial S_{w}}{\partial x} \frac{d P_{c}}{d S_{w}} \quad \text { (likewise for } y, z\right)
\end{aligned}
$$

Using this in equation (4), we obtain the Darcy flow of the water phase:

$$
\vec{u}_{w}=\left(\frac{\lambda_{w} \lambda_{o}}{\lambda_{o}+\lambda_{w}}\right) \frac{d P_{c}}{d S_{w}}\left(\frac{\partial S_{w}}{\partial x}, \frac{\partial S_{w}}{\partial y}, \frac{\partial S_{w}}{\partial z}+\kappa\right)
$$

Where $\kappa$ is a term due to gravity:

$$
\kappa=\frac{\left(\lambda_{o} \rho_{o}+\lambda_{w} \rho_{w}\right) g}{\lambda_{o}} \frac{1}{\frac{d P_{c}}{d S_{w}}}
$$

We assume the contribution from gravity to be negligible and set $\kappa=0$ in equation (12).

Inserting equation (12) into equation (2), we obtain a diffusion type of equation for the water saturation $S_{w}$ as a function of time and place.

$$
\frac{\partial}{\partial x}\left(D \frac{\partial S_{w}}{\partial x}\right)+\frac{\partial}{\partial y}\left(D \frac{\partial S_{w}}{\partial y}\right)+\frac{\partial}{\partial z}\left(D \frac{\partial S_{w}}{\partial z}\right)=\frac{\partial S_{w}}{\partial t}
$$

where $D$ is a diffusion coefficient given by:

$$
D\left(S_{w}\right)=-\frac{\lambda_{w} \lambda_{o}}{\Phi\left(\lambda_{o}+\lambda_{w}\right)} \frac{d P_{c}}{d S_{w}}
$$

Results of Bech et $a l^{2}$ and Beckner et al ${ }^{4}$ indicate that we may assume the diffusion coefficient $D$ to be constant. Then equation (14) reduces to a linear partial differential equation, which may be solved by analytic methods.

$$
D \nabla^{2} S_{w}=\frac{\partial S_{w}}{\partial t}
$$

A number of physical experiments may be modeled by this equation by imposing various boundary conditions. Typically, a constant boundary condition is adequate; e.g. setting $S_{w}=1-S_{\text {or }}$, on the faces of the imbibing rock. However, when capillary end-effects are important - as typical in laboratory experiments - it has been found ${ }^{2}$ that such effects can be modeled with good approximation by imposing an exponential boundary condition of the form $S_{w}=1-e^{-\beta t}$, where $\beta$ is an inverse time constant.

tThis assumption is experimentally justified by the results of Cuiec et al 5 . 


\section{THE IMBIBITION UPTAKE}

We describe the oil saturated block as a rectangular parallelepiped with side lengths $a, b, c$ (see Fig. 1). As the boundary condition of the imbibition equation, we assume the plane $x=0$ to be maintained at a water saturation of $\varphi(y, z, t)$, and all other faces to be kept at zero. The solution $S_{w}(x, y, z, t)$ is found in Carslaw and Jaeger ${ }^{1}$ - using Greens functions:

$$
\begin{aligned}
& S_{w}(x, y, z, t)= \\
& \frac{8 D \pi}{a^{2} b c} \sum_{l=1}^{\infty} \sum_{m=1}^{\infty} \sum_{n=1}^{\infty} l \sin \frac{l \pi x}{a} \sin \frac{m \pi y}{b} \sin \frac{n \pi z}{c} \\
& \times \int_{0}^{t} d \tau \int_{0}^{b} \int_{0}^{c} \sin \frac{m \pi y^{\prime}}{b} \sin \frac{n \pi z^{\prime}}{c} \varphi\left(y^{\prime}, z^{\prime}, \tau\right) \\
& \times \exp \left\{-D \pi^{2}(t-\tau)\left[\frac{l^{2}}{a^{2}}+\frac{m^{2}}{b^{2}}+\frac{n^{2}}{c^{2}}\right]\right\} d y^{\prime} d z^{\prime}
\end{aligned}
$$

If we assume that the water saturation on the boundary is a function of $z$ alone; i.e. $\varphi(y, z, t) \equiv \varphi(z, t)$, then the total volume uptake $U(t)$ of water in the block is calculated to:

$$
\begin{aligned}
& U(t) \equiv \Phi \int_{0}^{a} \int_{0}^{b} \int_{0}^{c} S_{w}(x, y, z, t) d x d y d z= \\
& \frac{128 \Phi D b}{\pi^{3} a} \sum_{l=0}^{\infty} \sum_{m=0}^{\infty} \sum_{n=0}^{\infty} \frac{1}{(2 m+1)^{2}(2 n+1)} \\
& \times \int_{0}^{t} \cdot\left[\int_{0}^{c} \sin \frac{(2 n+1) \pi z}{c} \varphi(z, \tau) d z\right] \\
& \times e^{-D \alpha_{l, m, n}(t-\tau)} d \tau
\end{aligned}
$$

where

$$
\begin{aligned}
& \alpha_{l, m, n}= \\
& \pi^{2}\left[\frac{(2 l+1)^{2}}{a^{2}}+\frac{(2 m+1)^{2}}{b^{2}}+\frac{(2 n+1)^{2}}{c^{2}}\right]
\end{aligned}
$$

In the Appendix we derive explicit solutions to this equation, imposing three different boundary conditions.

\section{MOVEMENT OF THE FREE WATER LEVEL}

Single Block System A single block/fracture system is modeled by a rectangular parallelepiped surrounded by a fracture volume $V_{f}$ (see Fig. 1). The water is injected into the fracture with imbibition occurring along the vertical sides of the matrix block only. The water level $\zeta(t)$ in the fracture system is determined from a volume balance consideration:

$$
Q_{w}(t)=Q_{f}+Q_{m}=\zeta(t) V_{f}+Q_{m}(t)
$$

where $Q_{w}$ is the cumulative water injected, and $Q_{f}, Q_{m}$ are the cumulative uptake of water in fracture and matrix block, respectively. The water level $\zeta(t)$ is measured relative to the block height.

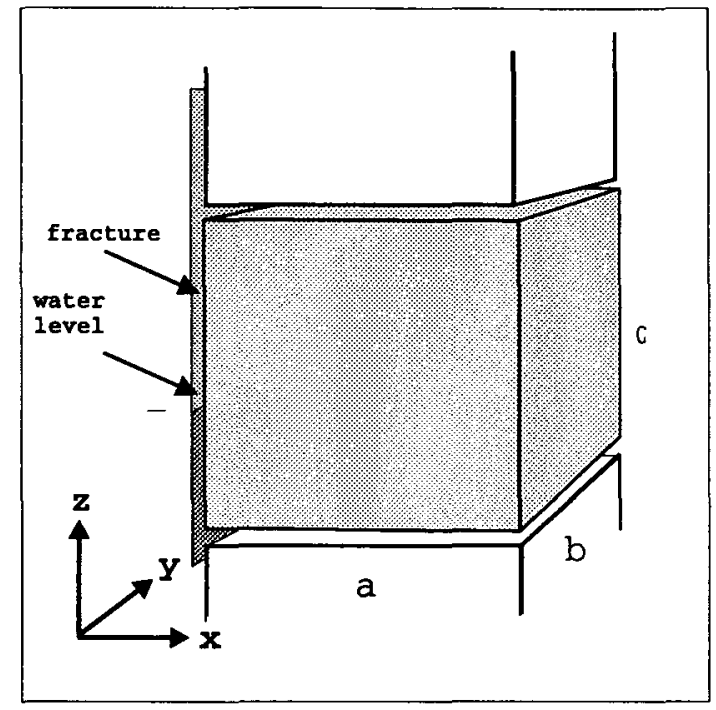

Figure 1: The fracture/block system

The water imbibition uptake can be found from equation (18) using the following saturation boundary function $\varphi(z, t)$ on each of the vertical faces:

$$
\varphi(z, t)= \begin{cases}1 & \text { if } z<c \zeta(t) \\ 0 & \text { otherwise }\end{cases}
$$

The total uptake $Q_{m}$ is the sum of contributions for each of the vertical faces with the above boundary condition:

$$
Q_{m}(t)=U(t)_{x=0}+U(t)_{y=0}+U(t)_{x=a}+U(t)_{y=b}
$$

The water uptake $U(t)_{x=0}$ due to imbibition from face $x=0$ is found by applying the boundary condition (21) into equation (18) and then calculating the resulting integral:

$$
\begin{aligned}
& \int_{0}^{\min (c, c \zeta)} \sin \frac{(2 n+1) \pi z}{c} d z \\
& =\frac{c}{(2 n+1) \pi}(1-\cos [(2 n+1) \pi \min (1, \zeta)])
\end{aligned}
$$

Since the water uptake from the face $y=0$ is found by interchanging $a$ with $b$ and $l$ with $m$ in equation (18) and since opposite faces have equal uptake, we find $Q_{m}$ as:

$$
\begin{aligned}
& Q_{m}(t)=2 \frac{128 \Phi D}{\pi^{4} a b c} \times \\
& \sum_{l=0}^{\infty} \sum_{m=0}^{\infty} \sum_{n=0}^{\infty} \frac{c^{2}}{(2 n+1)^{2}}\left(\frac{b^{2}}{(2 m+1)^{2}}+\frac{a^{2}}{(2 l+1)^{2}}\right) \times \\
& \int_{0}^{t}(1-\cos [(2 n+1) \pi \min (1, \zeta)]) e^{-D \alpha_{l, m, n}(t-\tau)} d \tau
\end{aligned}
$$

Rewriting equation (20) we find the height of the free water level as:

$$
\zeta(t)=\frac{Q_{w}(t)-Q_{m}(t, \zeta)}{V_{f}}
$$


This equation is solved by formulating the integral of equation (24) into a set of first order differential equations, which can be solved numerically. I.e. let $F_{l, m, n}(t)$ denote the integral of equation (24):

$$
\begin{aligned}
& F_{l, m, n}(t)=\int_{0}^{t}(1-\cos [(2 n+1) \pi \min (1, \zeta(\tau))]) \\
& \times e^{-D \alpha_{l, m, n}(t-\tau)} d \tau \quad \ldots \ldots \ldots \ldots \ldots \ldots \ldots \ldots \ldots \ldots \ldots \ldots
\end{aligned}
$$

From calculus we have the result:

$$
\begin{aligned}
& F(t) \equiv \int_{0}^{t} f(\tau, t) d \tau \Rightarrow \\
& \frac{d F}{d t}=f(t, t)+\int_{0}^{t} \frac{\partial}{\partial t} f(\tau, t) d \tau
\end{aligned}
$$

Using this on $F_{l, m, n}(t)$ we get:

$$
\begin{aligned}
& \frac{d F_{l, m, n}}{d t}= \\
& 1-\cos [(2 n+1) \pi \min (1, \zeta(t))]-D \alpha_{l, m, n} F_{l, m, n}(t)
\end{aligned}
$$

This equation, together with the constraint of equation (25), forms an infinite set of ordinary differential equations, which must be solved numerically.

Multiple Blocks System A large number of the above matrix/fracture systems may be combined to formulate a very simple fractured reservoir model. This model must take the impact of the increasingly larger volume of exposed matrix blocks into account as the free water level rises in the reservoir. Since only the vertical movement of the water level is considered, we restrict the block combination to a vertical stacking. Furthermore, we will assume that all the blocks are identical and that no water bearing fractures are separating the stacked blocks - i.e. no imbibition occurs at the top or bottom of the blocks. This latter assumption reflects the physical fact of vertical compaction of the fracture system due to the overburden. In the material balance consideration of equation (20) the contribution from the matrix block should now be considered as a sum of contributions from each exposed matrix block.

$$
\begin{gathered}
Q_{w}(t)=\zeta(t) V_{f}+Q_{m}^{0}(t)+Q_{m}^{1}(t)+Q_{m}^{2}(t)+ \\
\cdots+Q_{m}^{N}(t) \quad \ldots \ldots \ldots \ldots \ldots \ldots \ldots \ldots \ldots \ldots \ldots
\end{gathered}
$$

where we assume that $N$ blocks have been fully exposed to the water i.e. the water level has reached the $N+1$ 'th block $(N<\zeta<N+1)$. The total matrix uptake $Q_{m}(t)$ is found from the single block result in equation (24) as:

$$
\begin{aligned}
& Q_{m}(t)=Q_{m}^{0}(t)+Q_{m}^{1}(t)+Q_{m}^{2}(t) \cdots+Q_{m}^{N}(t)= \\
& 2 \frac{128 \Phi D}{\pi^{4} a b c} \sum_{l=0}^{\infty} \sum_{m=0}^{\infty} \sum_{n=0}^{\infty}\left(\frac{b^{2}}{(2 m+1)^{2}}+\frac{a^{2}}{(2 l+1)^{2}}\right) \\
& \times c^{2} \frac{F_{l, m, n}^{0}(t)+\cdots+F_{l, m, n}^{N}(t)}{(2 n+1)^{2}} \quad \ldots \ldots \ldots \ldots \ldots
\end{aligned}
$$

where the functions $F_{l, m, n}^{0}(t), \ldots, F_{l, m, n}^{N}(t)$ are defined as in equation (26) and obey a modified version of the differential equation (28):

$$
\begin{aligned}
& \frac{d F_{l, m, n}^{i}}{d t}=1-\cos [(2 n+1) \pi \min (1, \zeta(t)-i)] \\
& -D \alpha_{l, m, n} F_{l, m, n}^{i}(t) \quad \ldots \ldots \ldots \ldots \ldots \ldots \ldots \ldots \ldots \ldots
\end{aligned}
$$

For $i<N$ this reduces to: $2-D \alpha_{l, m, n} F_{l, m, n}^{i}(t)$. Hence, for $F_{l, m, n}(t)=F_{l, m, n}^{0}(t)+\cdots+F_{l, m, n}^{N}(t)$ we have :

$$
\begin{aligned}
& \frac{d F_{l, m, n}}{d t}=2 N+1-\cos [(2 n+1) \pi \min (1, \zeta-N)] \\
& -D \alpha_{l, m, n} F_{l, m, n} \quad \ldots \ldots \ldots \ldots \ldots \ldots \ldots \ldots \ldots \ldots \ldots \ldots
\end{aligned}
$$

This equation can be considered as a generalization of equation (28) to $N$ blocks exposed to water. Likewise, it is solved numerically.

\section{NUMERICAL RESULTS}

The numerical solution to the system of differential equations given by equation (32) and equation (29) is implemented in a $C$ program, and a fourth order RungeKutta solver is applied ${ }^{3}$. An investigation (Fig. 2) of the numerical convergence using different maxima of the indices $(l, n, m)$ in equation (30) showed a rapid convergence for the basic single block data in Table 1 . Therefore, a fixed maximum of $(l, m, n)=(10,10,10)$ for the studies in this section was applied.

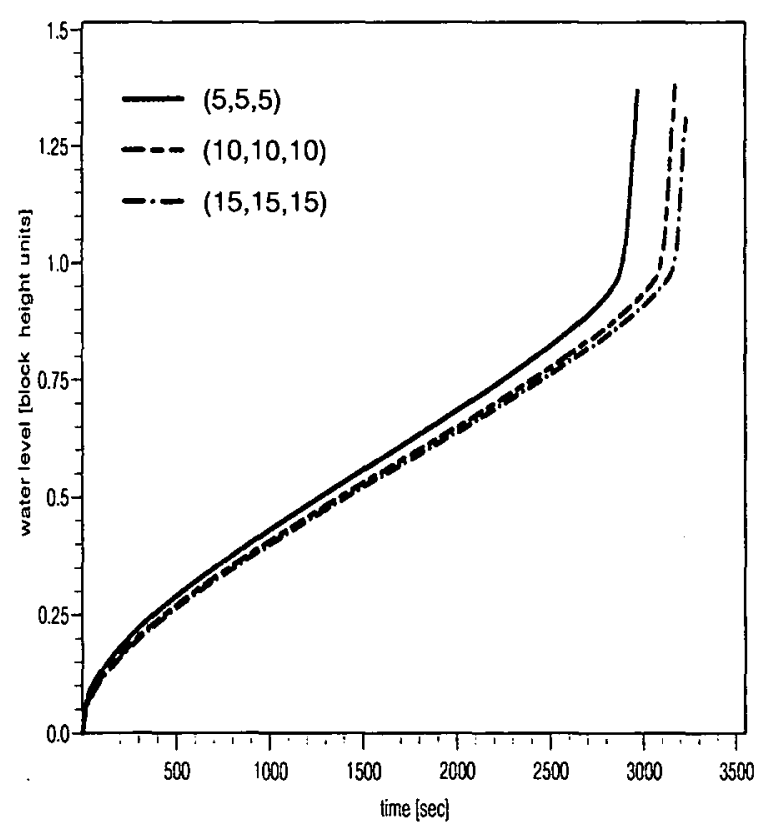

Figure 2: Investigation of numerical convergence using three sets of $(l, n, m)$

The basic data in Table 1 are derived from a modeling of the oil recovery curves from spontaneous imbibition in low-permeability chalk (Cuiec et al ${ }^{5}$ ). 
Table 1: Basic data for numerical studies.

\begin{tabular}{|ll|}
\hline Water injection rate $q_{w}$ & $=100 \mathrm{~cm}^{3} / \mathrm{s}$ \\
Fracture Volume $V_{f}$ & $=10000 \mathrm{~cm}^{3}$ \\
Block dimension $a, b, c$ & $=1 \mathrm{~m}, 1 \mathrm{~m}, 1 \mathrm{~m}$ \\
Diffusion Coefficient $D$ & $=100 \times 10^{-6} \mathrm{~m}^{2} / \mathrm{s}$ \\
Porosity $\Phi$ & $=50 \%$ \\
Fracture width $\left(\approx \frac{V_{f}}{2 c(a+b)}\right)$ & $=2.5 \mathrm{~mm}$ \\
\hline
\end{tabular}

Single Block Study. Three types of numerical investigations of the free water level movement in a single block matrix/fracture system were performed. The basic data for these numerical investigations are given in Table 1.

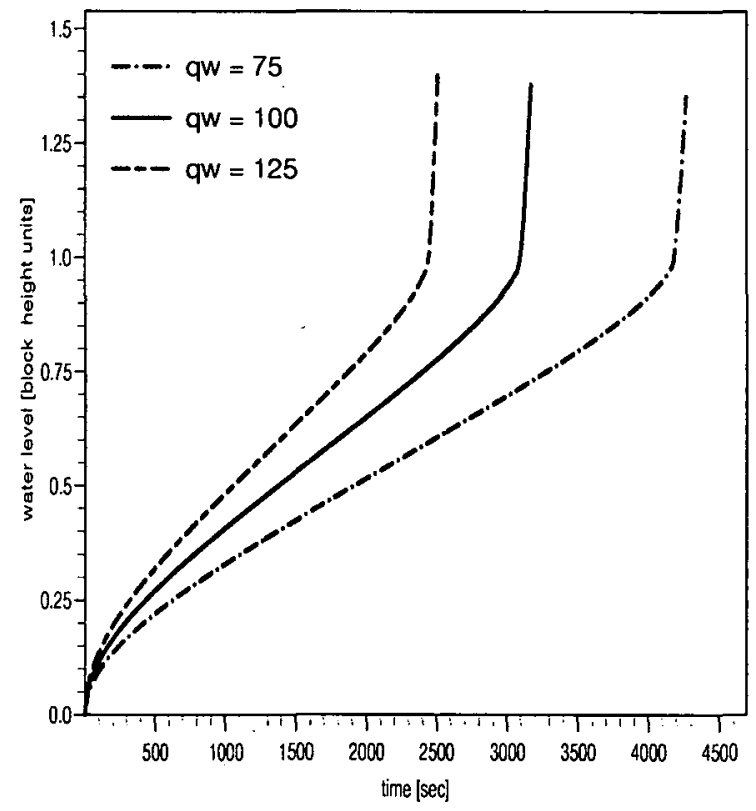

Figure 3: Single block, injection rate influence.

The first investigation considers the influence of different water injection rates. Fig. 3 gives the free water level height relative to the block height obtained for the three injection rates $75 \mathrm{~cm}^{3} / \mathrm{s}, 100 \mathrm{~cm}^{3} / \mathrm{s}$ and $125 \mathrm{~cm}^{3} / \mathrm{s}$, respectively $\left(6.48 \mathrm{~m}^{3} /\right.$ Day, $8.64 \mathrm{~m}^{3} /$ Day and $10.8 \mathrm{~m}^{3} /$ Day). In all cases a dominant linear behavior of the water level is observed for intermediate water levels. The non-linear behavior is more dominant for the high rate case. A constant fracture water level velocity is estimated from the linear part of the curves and found to $\approx 1.08 \mathrm{~cm} / \mathrm{min}, 1.44 \mathrm{~cm} / \mathrm{min}$ and $1.81 \mathrm{~cm} / \mathrm{min}$, respectively $(15.5 \mathrm{~m} /$ Day, $20.7 \mathrm{~m} /$ Day and $26.1 \mathrm{~m} /$ Day $)$. We observe an approximately linear relation between these fracture water level velocities and the injection rates.

In the second investigation we consider the influence of varying the diffusion coefficient. Fig. 4 gives the resulting curves for diffusion coefficients selected to $10 \cdot 10^{-6} \mathrm{~m}^{2} / \mathrm{s}, 100 \cdot 10^{-6} \mathrm{~m}^{2} / \mathrm{s}$ and $1000 \cdot 10^{-6} \mathrm{~m}^{2} / \mathrm{s}$, respectively. We observe that for the two high diffu-

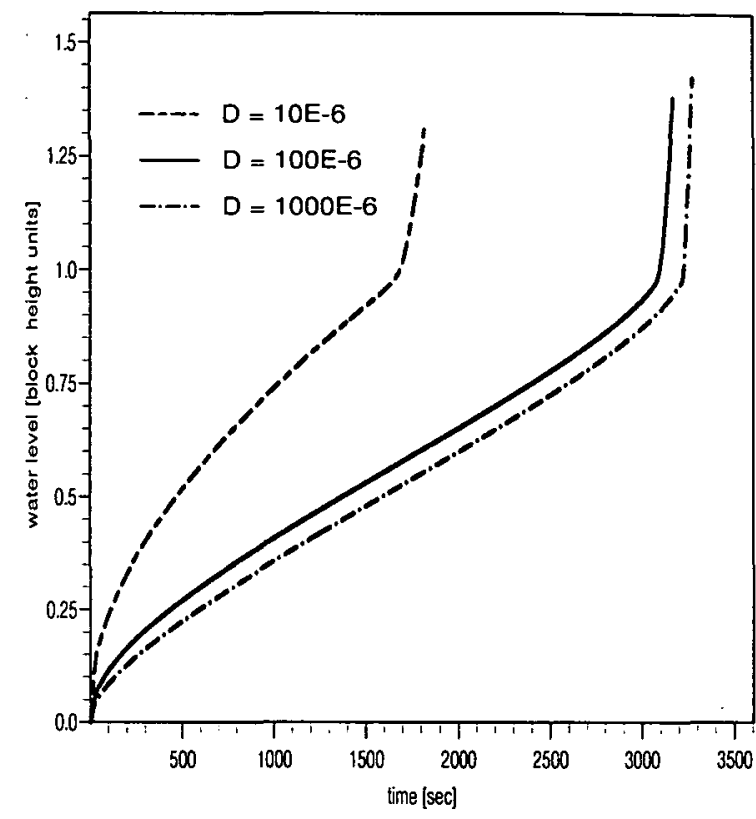

Figure 4: Single block, diffusion coefficient, influence.

sion values we get identical results except for the initial takeoff. The linear part of the curves has a slope of $\approx$ $1.43 \mathrm{~cm} / \mathrm{s}(20.6 \mathrm{~m} /$ Day $)$, whereas a linear part of the low diffusion curve is never obtained.

In the third investigation we consider the influence of the matrix block sidelength. We used blocks with identical sidelengths $(a=b=c)$ and selected lengths of $1,1.5$ and $2 \mathrm{~m}$ for the computations. Extracting the slopes from the linear part of the resulting curves in Fig. 5, we get relative fracture water level velocities as $\approx 1.44 \mathrm{~min}^{-1}, 0.43 \mathrm{~min}^{-1}$ and $0.18 \mathrm{~min}^{-1}$, respectively (20.7 $\mathrm{Day}^{-1}, 6.2 \mathrm{Day}^{-1}$ and 2.6 $\mathrm{Day}^{-1}$ ). We notice a linearity existing between these data and the inverse of the matrix block volumes.

In Fig. 4, it was found that identical results are obtained for the two high diffusion curves. This means that the diffusion capacity is capable of fully balancing the water injection rate. An increase in the diffusion coefficient will not effect the water velocity in the fracture system.

Vertically Stacked Blocks. For a system of vertically stacked blocks, numerical investigations similar to the single block studies above, were performed. The basic data in Table 1 for the identical blocks were used, and a total of 100 blocks were selected; i.e. $N<100$ in equation (32). The results are displayed in Fig. 6, Fig. 8 and Fig. 10, and in all cases a clear linear rise in the free water level is observed. In Fig. 6 we find fracture water level velocities of $\approx 1.40 \mathrm{~cm} / \mathrm{min}, 1.86$ $\mathrm{cm} / \mathrm{min}$ and $2.33 \mathrm{~cm} / \mathrm{min}$, respectively $(20.1 \mathrm{~m} /$ Day, $26.8 \mathrm{~m} /$ Day and $33.5 \mathrm{~m} /$ Day), and in Fig. 8 no effect of applying a different diffusion coefficient is observed, and the resulting curve gives a fracture water level velocity of $\approx 1.86 \mathrm{~cm} / \mathrm{min}(26.8 \mathrm{~m} /$ Day $)$. In Fig. 10 we find fracture water level velocities of $\approx 1.86 \mathrm{~cm} / \mathrm{min}, 0.85$ 


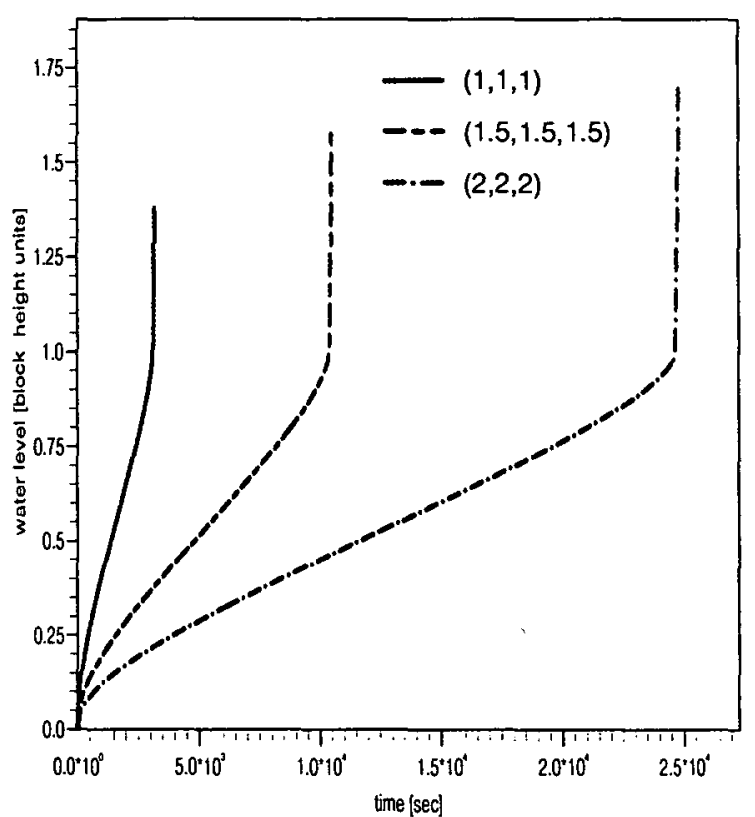

Figure 5: Single block, block size influence for three sets of $(a, b, c)$.

$\mathrm{cm} / \mathrm{min}$ and $0.47 \mathrm{~cm} / \mathrm{min}$ ( $26.8 \mathrm{~m} /$ Day, $12.2 \mathrm{~m} /$ Day and $6.8 \mathrm{~m} /$ Day). In all cases it is found that the slope can be derived, with good approximation, as an average slope of the corresponding single block study. This result holds in all cases where the diffusion capacity is capable of fully balancing the injected water. Hence, in these cases we may determine the fracture water level velocity of the water front in a stacked block/fracture system as the average velocity for a single block system.

In Fig. 7 the computed fracture water level velocity versus time for the low injection rate case is displayed. We observe that the dynamic behavior from the underlying single blocks is revealed by a large variation in the fracture water level velocities (15-50 m/Day) as new matrix block surfaces are contacted.

The effect of the vertical stacking on the diffusion capacity of the total matrix system is revealed in Fig. 9 , where the initial water level of the diffusion investigation in Fig. 8 is shown. For the low diffusion coefficient case we observe a gradual increase in the uptake capability as more and more blocks are exposed, and a clear linear behavior on a single block scale is found after just a few block heights. This observation, together with the discussion on Fig. 4 above, indicates that the velocity of the free water level in a fractured system does not depend on variation in the matrix blocks' capillary forces, but only on the geometry of the fracture system and the applied water injection rates.

We may therefore describe the large scale behavior of the system by a simple tank model, where matrix and fracture are coupled directly to the injected water. Such a model is entirely described by a volume balance consideration on the cumulative injected water volume

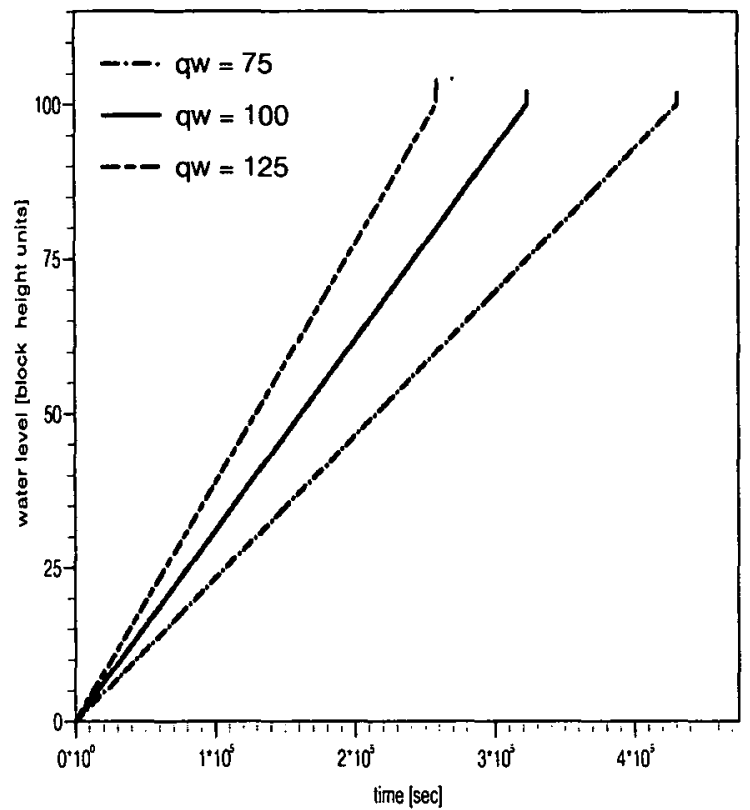

Figure 6: Stacked blocks, injection rate influence.

$Q_{w}:$

$$
Q_{w}=V_{f} \zeta+V_{m} \zeta
$$

Here $V_{f}$ and $V_{m}$ are the pore volumes of the fracture and matrix, respectively. Differentiating equation (33), we obtain the fracture water level velocity:

$$
\frac{d \zeta}{d t}=\frac{q_{w}}{V_{f}+V_{m}}
$$

Here $q_{w}$ is the applied constant injection rate. This equation yields fracture water level velocities of 1.32 $\mathrm{cm} / \mathrm{min}, 1.76 \mathrm{~cm} / \mathrm{min}$ and $2.21 \mathrm{~cm} / \mathrm{min}$, respectively ( $19.1 \mathrm{~m} /$ Day, $25.4 \mathrm{~m} /$ Day and $31.8 \mathrm{~m} /$ Day) for the three injection rates in Fig. 6. The results are seen to be in good agreement with the findings above (within $\approx 5 \%$ ). In applying equation (34) we have taken into account that not all matrix block sides are in contact with the water. In fact the imposed boundary condition for the stacked block system allows no water saturation at the top and bottom surfaces of the matrix blocks. Hence the volume $V_{m}$ of equation (34) has been multiplied by a factor of $4 / 6$.

\section{APPLICATION}

The mathematical model presented here has been implemented in a user-friendly graphical environment on an $\mathrm{X} / \mathrm{Windows}$ workstation. The program allows modeling of core plug laboratory imbibition experiments using equation (40) to estimate oil recovery curves together with display of the associated saturation maps. This provides a unique tool for physical understanding of the basic data that governs the single block imbibition process.

Large scale phenomena may also be studied by stochastically combining numerous matrix blocks according to 


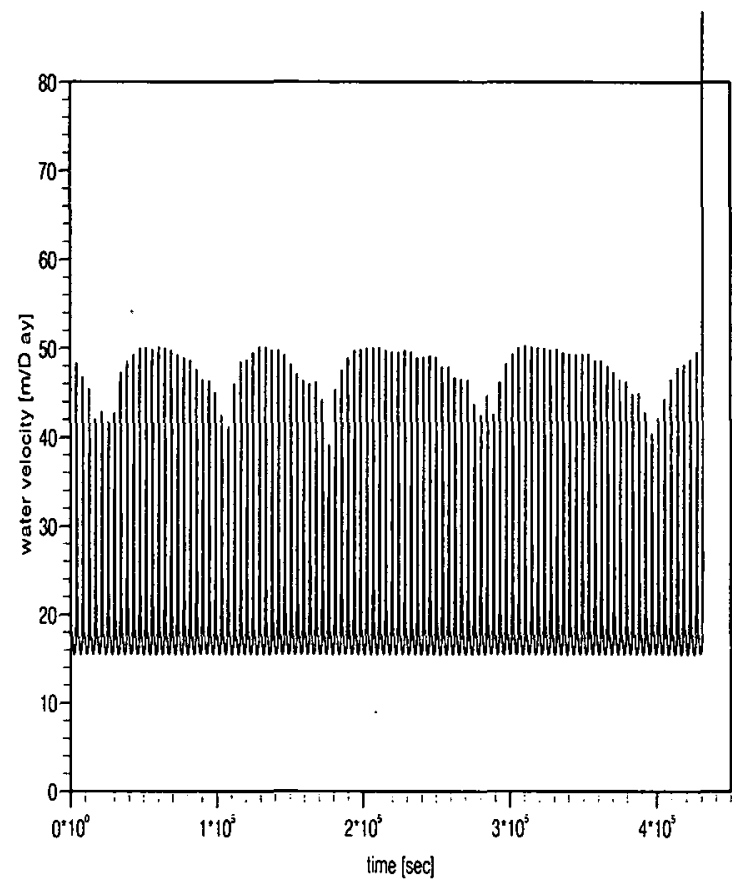

Figure 7: Stacked blocks low rate - fracture water level velocities.

a predescribed distribution function - allowing characteristic block geometries to dominate. Assuming the model completely submerged into water, recovery curves are established using equation (37). Such studies will determine the influence of the fracture geometry on the oil recovery profiles.

Vertical movement of the free water level is modeled either for a single block or a system of vertically stacked blocks using equation (32). This will allow an estimation of the expected fracture water velocity in a given fracture geometry subjected to water injection.

As a whole, the program serves as a valuable tool for defining the adequate input to a full field reservoir simulation model of a fractured field. The possibility of incorporating the model presented here directly in a reservoir simulator is also being considered.

\section{CONCLUSIONS}

This paper presents the derivation of a mathematical two-phase, 3D model that describes the vertical movement of the free water level in a fractured reservoir subjected to water injection. There are four specific results in this work.

1. We describe a simple self-contained mathematical model of the coupling between the fracture water level and the matrix block saturations. The model gives for low-permeability data a rapid convergence in the numerical solution methods.

2. Numerical investigations indicate that the vertical velocity of the free water level in a fractured reservoir does not depend on variation in the matrix blocks' capillary forces, but only on the geometry of the fracture system and the applied water injection rates.

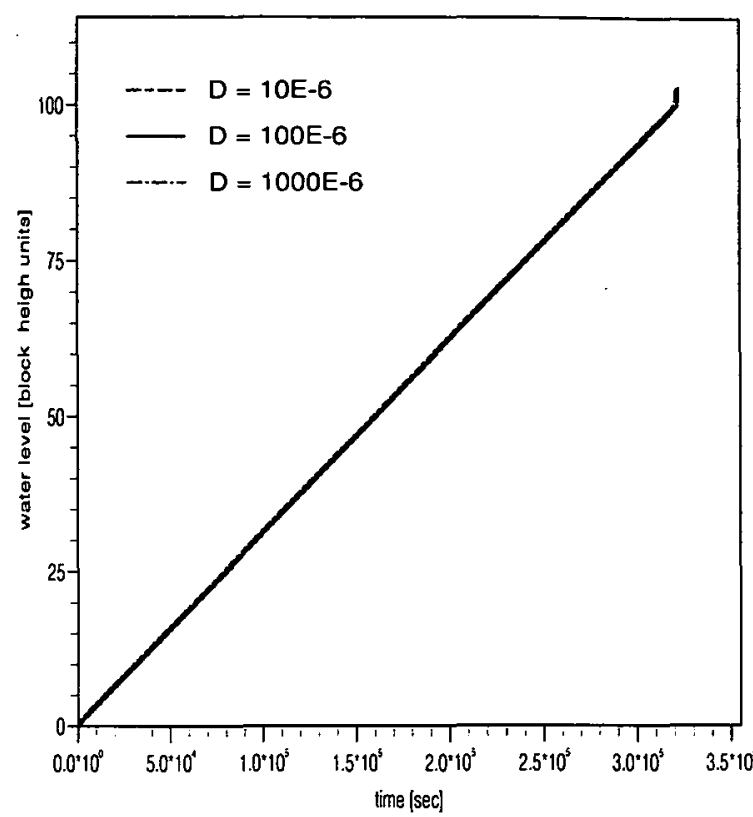

Figure 8: Stacked blocks, diffusion coefficient influence.

3 . The velocity of the vertical water movement in a simple fractured system can be derived from a single block/fracture model.

4. Results show a linear relation between the supplied injection rates, the inverse of the matrix block volumes and the velocity of the free water level. A simple equation based on a tank model was found to be in good agreement with the large scale behavior of the fracture/matrix system.

\section{NOMENCLATURE}

$a, b, c=$ block sidelengths $[\mathrm{m}]$

$S \quad=$ saturation [fraction]

$\vec{u} \quad=$ phase velocity $[\mathrm{m} / \mathrm{s}]$

$q=$ flow rate $\left[\mathrm{m}^{3} / \mathrm{s}\right]$

$P \quad=$ pressure $[\mathrm{pa}]$

$K=$ formation permeability $\left[\mathrm{m}^{2}\right]$

$k=$ relative permeability

$D=$ diffusion coefficient $\left[\mathrm{m}^{2} / \mathrm{s}\right]$

$Q=$ cumulative production $\left[\mathrm{m}^{3}\right]$

$t \quad=$ time [s]

$U \quad=$ uptake function $\left[\mathrm{m}^{3}\right]$

$V=$ volume $\left[\mathrm{m}^{3}\right]$

$F=$ integration function $[\mathrm{s}]$

$g=$ gravitational constant $\left[\mathrm{m} / \mathrm{s}^{2}\right]$

\section{Greek}

$\Phi=$ porosity [fraction]

$\rho=\operatorname{density}\left[\mathrm{kg} / \mathrm{m}^{3}\right]$

$\mu \quad=$ viscosity [pa s]

$\lambda \quad=$ mobility $\left[\mathrm{m}^{3} \mathrm{~s} / \mathrm{kg}\right]$

$\zeta=$ water level [block height units]

$\tau=$ time $[\mathrm{s}]$ 


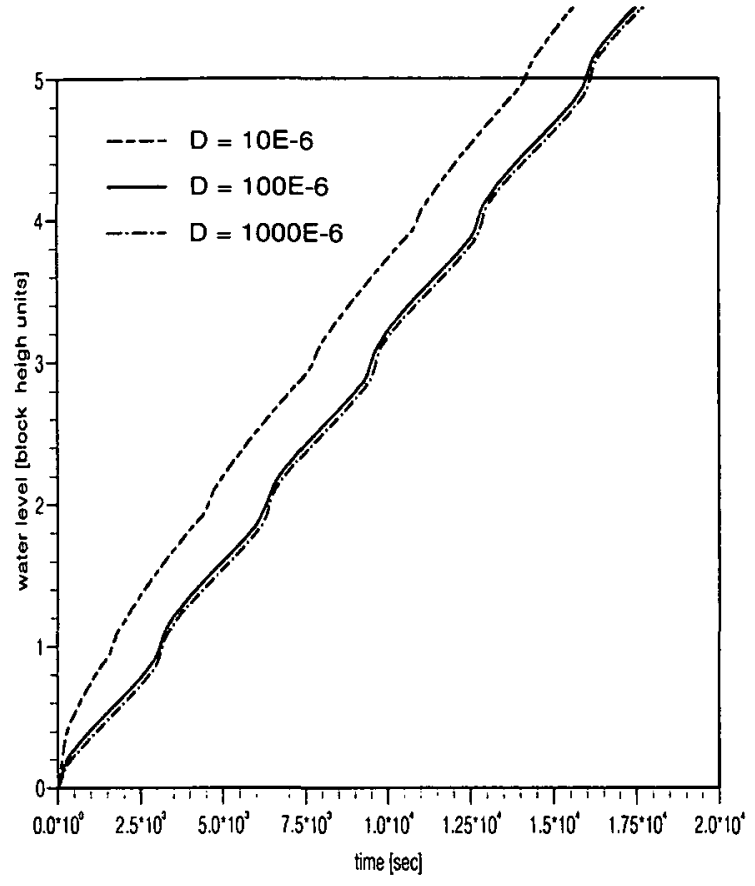

Figure 9: Stacked blocks, diffusion coefficient influence.

$\varphi \quad=$ saturation function [fraction]

\section{Subscripts}

$l, m, n=$ summation index

$m \quad=$ matrix

$f \quad=$ fracture

o $\quad$ oil

$w \quad=$ water

$j \quad=$ phase index; i.e. oil or water

\section{ACKNOWLEDGEMENTS}

Partial funding of this work by the Danish Ministry of Energy is gratefully acknowledged.

\section{REFERENCES}

1. H.S. Carslaw \& J.C. Jaeger "Conduction of Heat in Solids" Oxford University Press 1959, p. 362.

2. N. Bech, O.K. Jensen \& B. Nielsen: "Modeling of the Gravity Imbibition and Gravity Drainage Processes: Analytic and Numerical Solutions" SPE Reservoir Engineering, 1991.

3. W.H. Press, B.P. Flannery, S.A. Teukolsky \& W.T.Vetterling "Numerical Recipes - The Art of Scientific Computing" Cambridge University Press 1987.

4. B.L. Beckner, K. Ishimoto, S. Yamaguchi, A. Firoozabadi \& K. Aziz "Imbibition-D̃ominated Matrix-F̃racture Fluid Transfer in Dual Porosity Simulators" Paper SPE 16981, presented at the 1987 SPE Annual Technical Conference and Exhibition, Dallas, Sept. 27-30.

5. L. Cuiec, B. Bourbiaux \& F. Kalaydjian "Oil Recovery by Imbibition in Low-Permeability Chalk"

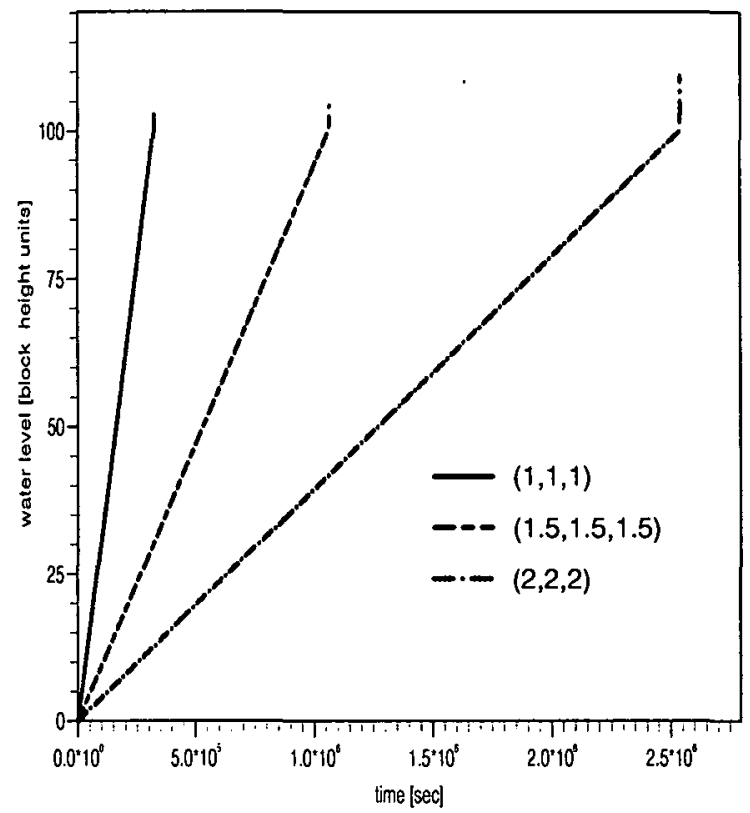

Figure 10: Stacked blocks, block size influence for three sets of $(a, b, c)$.

Paper SPE 20259, SPE Formation Evaluation, Sep. 1994, first presented at the 1990 SPE/DOE Symposium on Enhanced Oil Recovery in Tulsa, April 22-25.

\section{APPENDIX}

Here we derive explicit solutions to the imbibition uptake equation (18) with three different boundary equations.

Example 1. Assume the face $x=0$ fully exposed to water; i.e. $\varphi(z, t)=1$. Then calculating

$$
\int_{0}^{c} \sin \frac{(2 n+1) \pi z}{c} d z=\frac{2 c}{(2 n+1) \pi}
$$

and

$$
\int_{0}^{t} e^{-D \alpha_{l, m, n}(t-\tau)} d \tau=\frac{1}{D \alpha_{l, m, n}}\left(1-e^{-D \alpha_{l, m, n} t}\right)
$$

we find the total uptake $U_{1}(t)$ as:

$$
\begin{aligned}
& U_{1}(t)=\frac{256 \Phi D}{\pi^{4}} \frac{b c}{a} \times \\
& \sum_{l=0}^{\infty} \sum_{m=0}^{\infty} \sum_{n=0}^{\infty} \frac{1-e^{-D \alpha_{l, m, n} t}}{D \alpha_{l, m, n}(2 m+1)^{2}(2 n+1)^{2}}
\end{aligned}
$$

Example 2. Assume the face $x=0$ partly exposed to water to a level $\zeta$; i.e.

$$
\varphi(z, t)= \begin{cases}1 & \text { if } z<\zeta c ; \zeta<1 \\ 0 & \text { otherwise }\end{cases}
$$

Then calculating

$$
\int_{0}^{\zeta c} \sin \frac{(2 n+1) \pi z}{c} d z=\frac{c}{(2 n+1) \pi}(1-\cos [(2 n+1) \pi \zeta])
$$


we find the total uptake $U_{2}(t)$ as:

$$
\begin{aligned}
& U_{2}(t)=\frac{128 \Phi D}{\pi^{4}} \frac{b c}{a} \times \\
& \sum_{l=0}^{\infty} \sum_{m=0}^{\infty} \sum_{n=0}^{\infty} \frac{(1-\cos [(2 n+1) \pi \zeta])\left(1-e^{-D \alpha_{l, m, n} t}\right)}{D \alpha_{l, m, n}(2 m+1)^{2}(2 n+1)^{2}}
\end{aligned}
$$

Example 3. Assume a constant rising water level along the face $x=0$ :

$$
\varphi(z, t)= \begin{cases}1 & \text { if } z<v t ; v>0 \\ 0 & \text { otherwise }\end{cases}
$$

Calculating the integral of equation (18):

$$
\begin{aligned}
& \int_{0}^{t}\left(\int_{0}^{\min (c, v \tau)} \sin \frac{(2 n+1) \pi z}{c} d z\right) e^{-D \alpha_{l, m, n}(t-\tau)} d \tau \\
& =\frac{c}{(2 n+1) \pi} \times \\
& \int_{0}^{t}\left(1-\cos \left[(2 n+1) \pi \min \left(1, \frac{v \tau}{c}\right)\right] e^{-D \alpha_{l, m, n}(t-\tau)} d \tau\right. \\
& =\frac{c}{(2 n+1) \pi} \times \\
& {\left[\int_{0}^{t}\left(1-\cos \frac{(2 n+1) \pi v \tau}{c}\right) e^{-D \alpha_{l, m, n}(t-\tau)} d \tau\right.} \\
& \left.+\delta_{t} \int_{t_{o}}^{t} 2 e^{-D \alpha_{l, m, n}(t-\tau)} d \tau\right] \\
& =\frac{c}{(2 n+1) \pi}\left[e ^ { - D \alpha _ { l , m , n } ( t - \tilde { t } ) } \left(\frac{1-e^{-D \alpha_{l, m, n} \tilde{t}}}{D \alpha_{l, m, n}}-\right.\right. \\
& \frac{\left.D \alpha_{l, m, n} \cos \left(\omega_{n} t\right)+\omega_{n} \sin \left(\omega_{n} t\right)-e^{-D \alpha_{l, m, n} \tilde{t}} D \alpha_{l, m, n}\right\}}{\omega_{n}^{2}+D^{2} \alpha^{2}} \\
& \left.+\delta_{t} \frac{2}{D \alpha_{l, m, n}}\left\{1-e^{-D \alpha_{l, m, n}\left(t-t_{\circ}\right)}\right\}\right]
\end{aligned}
$$

Where $\omega_{n}=\frac{(2 n+1) \pi v}{c}, t_{0}=c / v, \tilde{t}=\min \left(t, t_{0}\right)$ and $\delta_{t}=\left\{\begin{array}{ll}1 & \text { if } t>t_{o} \\ 0 & \text { otherwise }\end{array}\right.$. This result inserted into equation (18) will yield the desired uptake $U_{3}(t)$. 


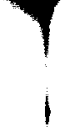

422 